\title{
A Suspended Plate In-Plane Resonator for Rheological Measurements at Tunable Frequencies
}

\author{
Martin Heinisch, Erwin K. Reichel, Bernhard Jakoby \\ Institute for Microelectronics and Microsensors \\ Johannes Kepler University \\ Altenbergerstr. 69 \\ 4040 Linz, Austria
}

\begin{abstract}
A resonating sensor for viscosity facilitating measurements at tunable frequencies is presented. A sample liquid is subjected to time harmonic shear stress induced by a vibrating plate. By measuring the resonant behavior of this fluid-structure interaction the liquid's rheological properties can be deduced. The resonating plate is affixed to two parallel wires, placed in an external static magnetic field. One of these wires is used to excite lateral vibrations of the plate by means of Lorentz forces while the second acts as pick-up providing an induced voltage representing the movement of the plate. The recorded frequency response is used to determine the liquid's physical properties. This setup, based on the mechanical coupling of two wires with a small plate and inducing time harmonic shear stresses with the latter, allows examining the test liquid at adjustable frequencies. In first experiments resonance frequencies from $820 \mathrm{~Hz}$ to $4040 \mathrm{~Hz}$ were achieved for a given geometry.
\end{abstract}

\section{Introduction}

The focus of our work is on tunable resonating rheometers. One advantage of resonating rheometers in comparison to conventional shear flow based rheometers is the possibility to characterize the physical properties of very complex non-newtonian liquids such as polymeric fluids, suspensions, emulsions etc. Vibrating viscometers are mechanically simpler than other types and the volume of fluid required for their use is much smaller making operation at high pressures and temperatures easier [1]. An overview of conventional principles for measuring viscosity is given in [2]. Piezoelectric or quartz crystal based oscillators proved to be well suitable for viscosity sensing and are commonly reported in literature, see e.g., [3], [4] and [5]. However, there is an increasing interest for vibrating resonators in the low kilohertz range to increase the penetration depth of the shear waves imposed into the liquid by the resonator [6], [7].

To determine the physical properties of a liquid such as viscosity or mass density using a resonator based principle, the change of the oscillator's quality factor and the shift of its resonant frequency (which are both known in vacuum or air) are recorded when it is immersed into the test liquid. For the investigation of some liquids it can either be important to analyze the liquid's physical behavior at one particular frequency or at several frequencies in a certain bandwidth. In both cases the capability of tuning the sensor's resonance frequency is required. In this contribution a rheometer based on a suspended in-plane vibrating plate with tunable resonance frequencies is presented. The principle of this sensor is depicted in Fig. 1(a). A similar setup has been presented in [7] aiming to improve the quality factor of micro-machined sensors. There, the suspended plate has been implemented in silicon technology. The sensor presented in this work consists of a non-conductive plate and two conductive wires placed in a constant magnetic field. The plate (for first prototypes PET and glass have been used) is affixed to the wires using thermal fusing or gluing, thus yielding a mechanical coupling of the two wires. One of these wires is used to deflect the plate in lateral direction by means of Lorentz forces on AC-currents flowing through the wire. The other wire, following the movement of the first, is used as pick-up representing the in-plane movement of the plate by an induced voltage. By varying the normal stresses within both wires by an appropriate tensioning mechanism the desired resonance frequency can be adjusted.

\section{Theory and Modeling}

\section{One-dimensional shear waves in viscous liquids}

To analytically describe the propagation of shear waves in a viscous liquid induced by a plate resonating at an angular frequency $\omega$ and amplitude $\hat{x}$ and considering a no-slip boundary condition at the solid-liquid interface, see Fig. 2(a), the stress tensor for liquids [8]

$$
\mathbf{T}=-p \mathbf{I}+2 \mu \mathbf{E}+\lambda \nabla \cdot \boldsymbol{u} \mathbf{I}
$$




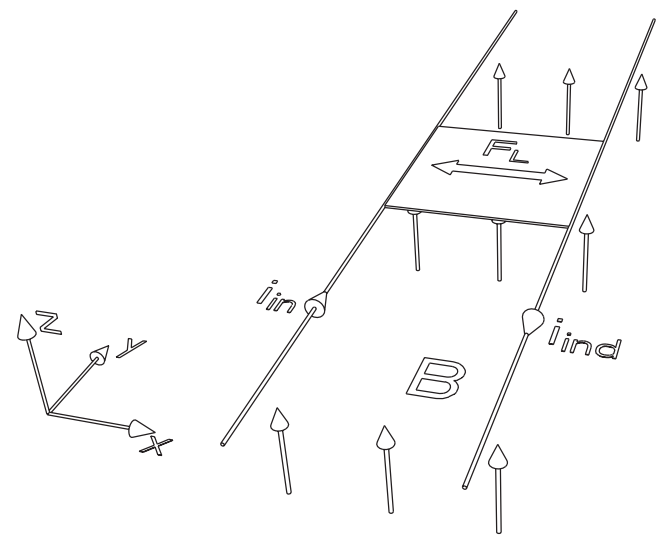

(a) Sensor Principle

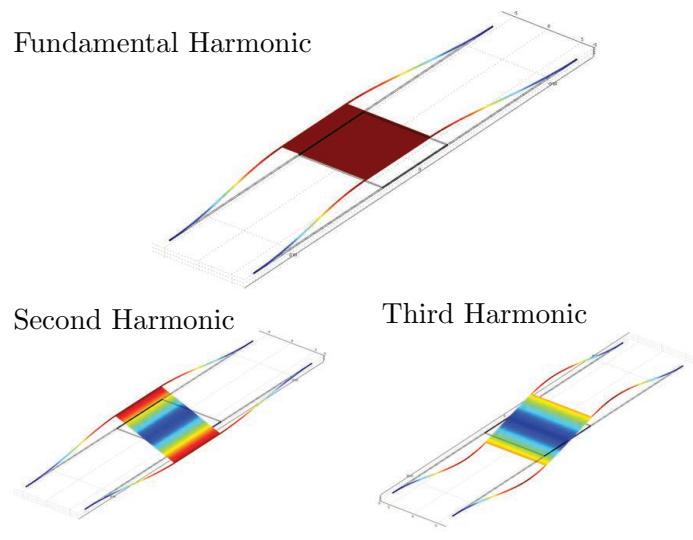

(b) FE-Simulations

Figure 1: In (a) the a schematic drawing of the suspended plate rheometer is depicted. The sensor consists of two parallel wires placed in external field $\mathbf{B}$. In this figure, the left wire is used for exciting lateral vibrations by means of Lorentz forces. The second wire on the right used for pick-up, is coupled to the excitation wire with a rigid plate, thus following the movement of the left wire by what a voltage is induced which is used as read-out. By changing the normal stresses in the wires, the resonance frequency can be changed. In (b) the first three modes (at resonance) are illustrated.

( $p$ : pressure, I: identity matrix, $\mu$ : dynamic viscostiy, $\mathbf{E}$ : symmetric part of tensor of deformation velocity, $\lambda$ : volume viscosity, $\boldsymbol{u}$ : deformation) is substituted into the linearized equation of motion [6]

$$
\rho \frac{\partial^{2} \boldsymbol{u}}{\partial t^{2}}=\nabla_{\mathrm{S}}^{\mathrm{T}} \mathbf{T}+\boldsymbol{f}_{\mathrm{V}}
$$

( $\rho$ : mass density, $\nabla_{\mathrm{S}}^{\mathrm{T}}$ : symmetric gradient operator, $\boldsymbol{f}_{\mathrm{V}}$ : volume forces e.g., gravitational force) which then has to be solved for $\boldsymbol{u}$.

Under the assumptions (i) that the infinitely extended plate is oscillating in $x$-direction (thus imposing only shear stress in $x$-direction), (ii) that gravitational forces are negligible, and (iii) that the liquid is incompressible, Eqs. 1 and 2 can be drastically simplified to the one dimensional problem

$$
\rho \frac{\partial^{2} u_{x}}{\partial t^{2}}=\frac{\partial T}{\partial z} \quad \text { with } \quad T=\mu \frac{\partial^{2} u_{x}}{\partial z \partial t} .
$$

Substituting $T$ in the equation of motion, transforming the problem to the frequency domain assuming a time dependence $e^{j \omega t}$, with $j=\sqrt{-1}$

$$
\frac{\partial}{\partial t} \circ \bullet j \omega \quad \text { and } \quad \frac{\partial^{2}}{\partial t^{2}} \circ \bullet-\omega^{2}
$$

and solving the linear differential equation of second order yields the solution for the deformation in $x$-direction

$$
u_{x}(z, t)=\hat{x} e^{-\frac{z}{\delta}} e^{-j\left(\frac{z}{\delta}-\omega t\right)}
$$

where

$$
\delta=\sqrt{\frac{2 \mu}{\rho \omega}}
$$

is the so-called penetration depth [6], [7] or decay length [3]. In Fig. 2(a) several solutions of a propagated shear wave during one period of oscillation are depicted.

\section{Lumped element model}

The scheme of the electrical equivalent circuit of the in-plane resonator consists of two galvanically isolated circuits, see Fig. 2(b). The input circuit on the left consists of a function generator with an output resistance of $50 \Omega$ connected to the excitation wire represented by a resistance $R_{1}$. The output circuit is modeled by two voltage sources $V_{\mathrm{M}}$ and $V_{\mathrm{ct}}$ accounting for motion induced voltage and electrical cross-talk, the resistance of the 


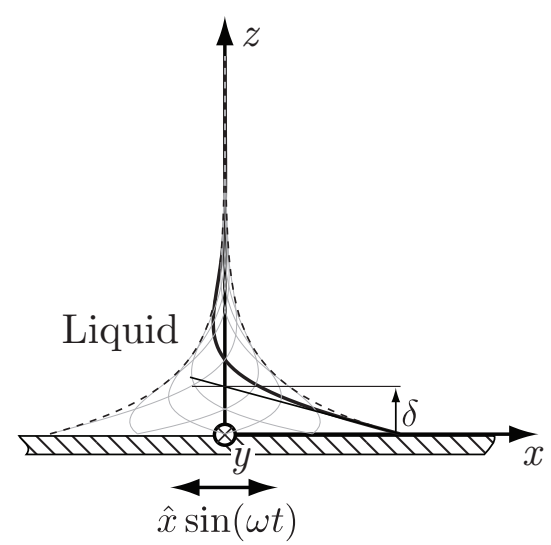

(a) Shear wave propagation
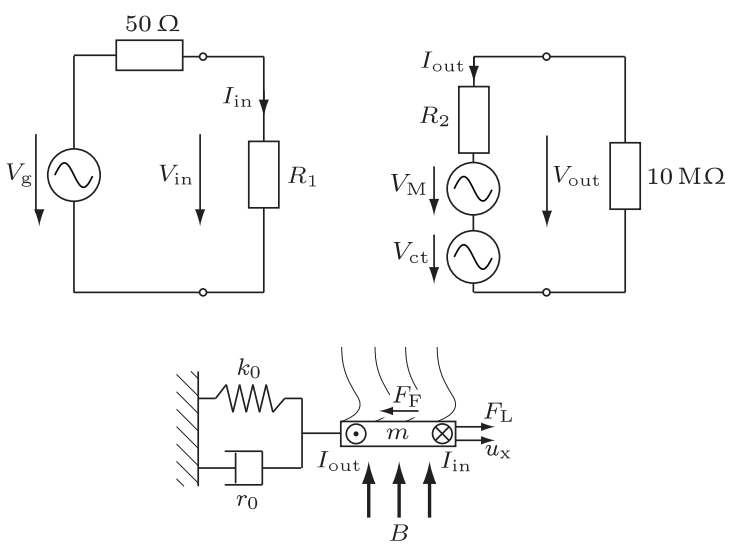

(b) El. equivalent circuit and mechanical lumped element model

Figure 2: In (a) the propagation of one-dimensional shear waves in a liquid induced by an oscillating plate with an amplitude $\hat{x}$ and angular frequency $\omega$ is illustrated. $\delta$ is the decay length. In (b) the scheme of the modeling approach is depicted. The upper figure shows the electrical equivalent circuit consisting of two galvanically isolated circuits representing the excitation and read-out circuits. $\left(V_{\mathrm{g}}\right.$ : Voltage of the function generator, $50 \Omega$ : Internal resistance of the function generator, $R_{1}$ : resistance of the excitation wire, $R_{2}$ : resistance of the pick-up wire, $V_{\mathrm{M}}$ : Motion induced voltage, $V_{\mathrm{ct}}$ : Induced voltage trough electrical cross-talk, $10 \mathrm{M} \Omega$ : Input resistance of the lock-in amplifier, $I_{\text {in }}, I_{\text {out }}, V_{\text {in }}, V_{\text {out }}$ Input and output currents / voltages ) The lower figure shows the mechanical lumped-element model. ( $k_{0}$ : intrinsic spring constant, $r_{0}$ : intrinsic damping parameter, $F_{\mathrm{F}}$ : Fluid forces, $F_{\mathrm{L}}$ : Lorentz forces, $u_{\mathrm{x}}$ : deformation in $x$-direction, $B$ : external magnetic field)

pick-up wire $R_{2}$ and the input-resistance of $10 \mathrm{M} \Omega$ of the lock-in amplifier. Note, that in this simplified model, the effect of the (mutual) inductance and motion induction in the excitation wire are neglected.

For modeling the damped in-plane oscillation, a linear spring-mass-damper oscillator, see Fig. 2(b) is used to describe the oscillator's intrinsic resonant behavior (in air) for the fundamental harmonic i.e., lateral oscillations in $x$-direction. The influence of the fluid on the oscillation is taken into account by considering a no-slip boundary condition at the platelet-liquid interface and assuming a one-dimensional shear wave propagation on both sides of the platelet. As the platelet's lateral dimensions are much larger than its thickness and as its surface $A$ is much larger than the wires surfaces, side effects emerging from displacement of the liquid at the platelets front sides as well as the flow around the wires are not taken into account. Thus, the fluid forces on the platelet are

$$
F_{\mathrm{F}}=-2 T(z=0) A
$$

where the shear stress

$$
T(z=0)=\hat{x}(1-j) \sqrt{\frac{\mu \rho \omega^{3}}{2}}
$$

is calculated by substituting Eqs. 5 and 6 into the the shear stress $T$ in Eq. 3 and evaluating for $z=0$.

With this, the transfer function of the mechanical oscillator, which is the quotient of displacement in $x$-direction $u_{\mathrm{x}}$ and Lorentz forces $F_{\mathrm{L}}=I_{\text {in }} B l^{*}$, where $I_{\text {in }}$ is the sinusoidal electrical current in the wire, $B$ is the magnetic flux density and $l^{*}$ is the wire's effective length (which depends on the mode shape but is in the order of the actual length) can be written as

$$
G_{\mathrm{m}}=\frac{u_{\mathrm{x}}}{F_{\mathrm{L}}}=\frac{1}{-\omega^{2}\left(m+A \sqrt{\frac{\mu \rho}{2 \omega}}\right)+j \omega\left(r_{0}+A \sqrt{\frac{\mu \rho \omega}{2}}\right)+k_{0}}
$$

Finally the motion-induced voltage in the pickup wire (coupled to the movement of the excitation wire) can be written as

$$
V_{\mathrm{M}}=j \omega G_{\mathrm{m}} \frac{B^{2} l^{* 2}}{R_{1}} V_{\text {in }}
$$

Depending on the wiring for power supply and the sensing of the induced voltage, it may be necessary to consider the effect of electrical cross-talk in the measured voltage as well, which can be easily taken into account with

$$
V_{\mathrm{ct}}=\frac{j \omega M}{R_{1}} V_{\mathrm{in}}
$$




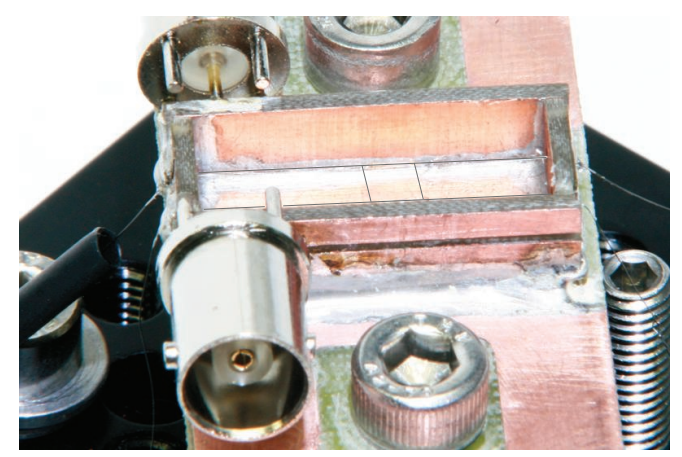

(a) Experimental well

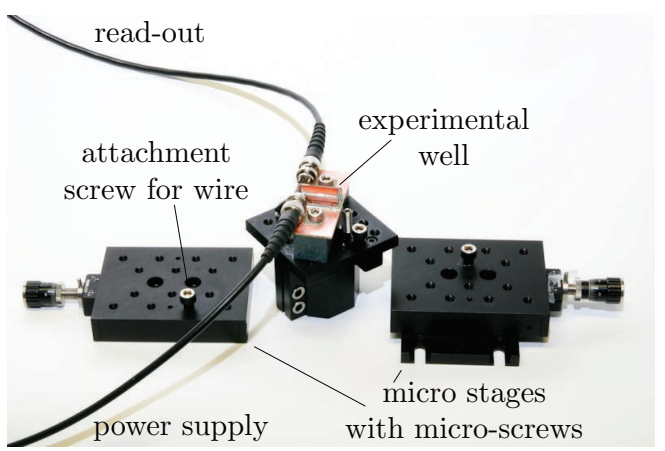

(b) Experimental Setup

Figure 3: In (a) a detailed view of the experimental well with the suspended plate sensor is illustrated. For better visibility, the platelet and the wires were shaded. The well can be filled with a liquid to be examined. To reduce electrical cross-talk and to keep the remaining cross-talk constant, BNC-connectors are used to keep the cross-sectional area of the loops which are formed by the excitation and pick-up wire with the external wiring as small as possible and constant. In (b) the experimental setup is illustrated. Two micro stages with micrometer screws are used to change the normal stresses in the wires to change the resonance frequency.

where $M$ is the mutual inductance describing the inductive coupling between excitation and read-out circuit. As the the input resistance of the lock-in amplifier is $10 \mathrm{M} \Omega$ it is assumed that $I_{\text {out }} \approx 0$ and thus

$$
V_{\text {out }}=V_{\mathrm{M}}+V_{\mathrm{ct}}
$$

\section{Experimental Setup}

\section{Sensor fabrication}

The wires for the sensor investigated for this contribution are $100 \mu \mathrm{m}$ tungsten wires. Tungsten features a high yield stress (about $550 \mathrm{MPa}$ ) as well as a high Youngs Modulus (about $11 \mathrm{GPa}$ ). (For a high tunable range by means of changing the normal stresses in a wire the yield stress should be preferentially high, whereas the Youngs modulus should be small.) For fabrication, the two wires were prestressed in parallel with a distance of about $4 \mathrm{~mm}$. Afterwards, a PET platelet of about $5 \times 5 \mathrm{~mm}$ was placed in the middle of both wires. The platelet was affixed to the wires by thermal fusion by applying an electrical current of about $2 \mathrm{~A}$ for a short time to the wires and thus fusing the PET platelet exactly at the location of the wires. As an alternative, the usage of glass platelets was also investigated. The higher mass density of glass compared to PET and the additional mass of the glue (epoxy resin was used) necessary to bond the glass platelet with the wires, yielded lower resonance frequencies at comparable equal normal stresses in the wires.

For many applications, materials such as glass will be the preferred material as it is proof to many aggressive liquids. However, gluing should be avoided first, as it hardly allows a reliable fabrication process, second, gluing beads change the shape of the flat surface, and third most glues are not compatible with aggressive liquids [9].

\section{Measuring setup}

The whole setup was mounted on an optic plate. For this, the sensor was put in a small experimental well for examining the resonant behavior in different liquids, see Fig. 3(a). The wires were attached to micro-stages with micrometer screws for changing the normal stresses in the wires. The vibrating length of the wires was determined by the mechanical contacts at the experimental well which served as electrical contacts as well. These electrical contacts were connected to BNC-connectors with as short as possible electrical paths and thus with a significantly reduced area which is relevant for the mutual inductance to avoid electrical cross-talk. The resonant behavior of the sensor was investigated by exciting it to time harmonic oscillations with a function generator, sweeping the frequencies within the band width of interest and recording the induced voltage in the pick-up wire with a lock-in amplifier.

\section{Measurements and Results}

\section{Electrical cross-talk}

In first measurements electrical cross-talk was observed, see Fig. 4(a). In air this electrical cross-talk does not have a significant effect on the signal close to the fundamental harmonic. However, when measuring liquids, this 

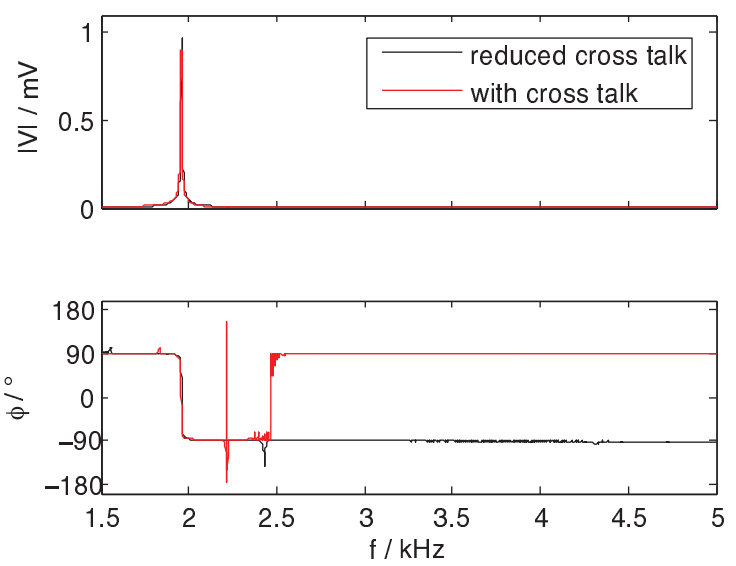

(a) Frequency response with and without cross-talk
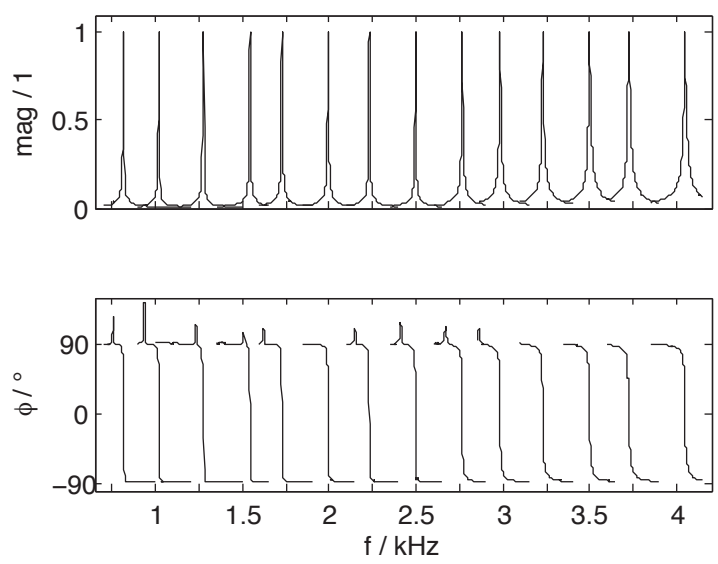

(b) Tuning of the resonance frequency

Figure 4: In (a) a comparison of the read-out signals with and with reduced crosstalk (in air) is shown. The difference in the measurements was that in case of 'reduced cross-talk' BNC-connectors were used. Where in air the effect of the cross-talk hardly influences the near resonant behavior of the sensor, it can become disturbing when examining liquids. In (b) resonance frequencies from $820 \mathrm{~Hz}$ up to $4040 \mathrm{~Hz}$ are illustrated. For changing the resonance frequencies for these measurements the normal stresses in the wires were varied by tensioning the wires with micro-stages with micrometer screws.

effect can become disturbing. The wires of the sensor connected to the wires for power supply and measuring form two loops (single-turn coils) with a certain cross-sectional area. The magnetic flux effected by the current in the first loop penetrating the second loop induces a voltage in this second loop (law of induction, third Maxwell equation) and vice versa. Displacing the wires changes the cross-sectional areas of the loops and thus, resulting in a different voltage $V_{\text {ct }}$, Eq. 11, making reliable measuring difficult. To reduce the effect of electrical cross-talk, BNC-connectors were brazed to the experimental well, first, to minimize the effective cross-sectional area of the single-turn loops and second to keep this cross-sectional area constant, see Fig. 3(a), enabling reliable measuring with strongly reduced electrical cross-talk, see Fig. 4(a).

\section{Tuning of the resonance frequency}

To show the capability of the tuning of the resonance frequency the normal stresses in the wires were changed by tensioning the wires with micro stages with micrometer screws. In a first experiment in air resonances between $820 \mathrm{~Hz}$ and $4040 \mathrm{~Hz}$ were achieved, see Fig. 4(b).

\section{Measurements in liquids}

The effect of liquids on the resonant behavior was investigated by measuring four different liquids, acetone, methanol, isopropanol and Di-water, see Fig. 5(a). The indicated values for viscosity and mass-density were measured with a Stabinger Viscometer (SVM 3000) at $25^{\circ} \mathrm{C}$. For all liquids, viscosity decreases with increasing temperature [10]. Thus, it has to be pointed out that it is always necessary to indicate the temperature prevailing during the measurement of viscosity. An overview of models describing the temperature depending decrease in viscosity is given in [11]. At the same time the intrinsic temperature dependence of the device characteristics may become relevant and need to be calibrated (which goes beyond the scope of this contribution, see, e.g., related discussion [12].) The model from Eq. 10 allows very accurate fitting, see Fig. 5(b), however based on our knowledge, comprehensive measurements have to be performed to determine the sensors intrinsic parameters to allow accurate and reliable sensing.

\section{Conclusion and Outlook}

This work shows the feasibility of measuring viscosity with this suspended-plate sensor at different resonance frequencies. Regarding future work, first of all a procedure to determine or predict the sensors intrinsic parameters at different environmental condition such as external temperature or pressure has to be developed. Extensive measurements have to be done to evaluate and estimate the achievable accuracies of this sensor principle. The applicability of the sensor on complex liquids such as viscoelastic liquids should be investigated as well. For a more accurate modeling, it might become important to model the wires as well. For this a similar approach like in [13] and [14] could be used. 

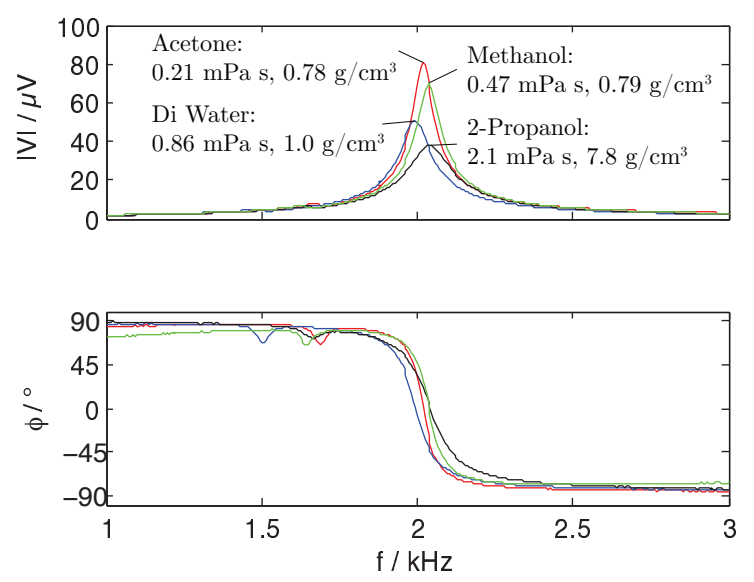

(a) Frequency response for four different liquids
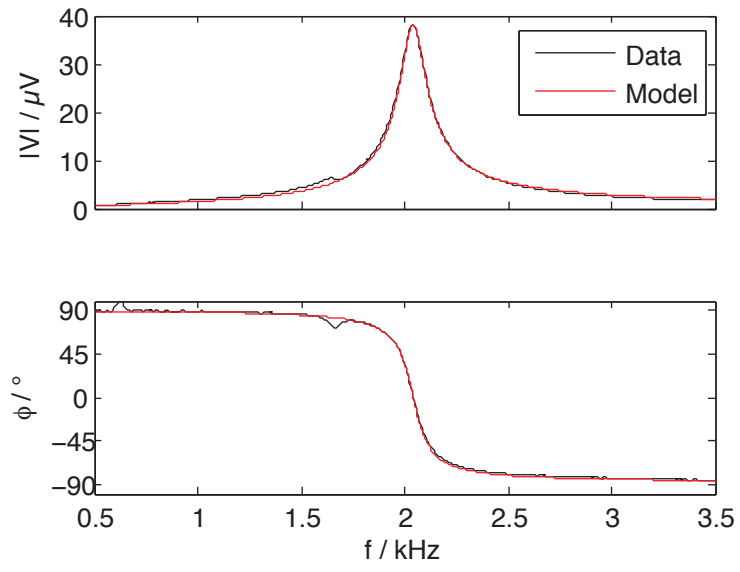

(b) Comparison model with data

Figure 5: In (a) the frequency response of the sensor including the fundamental harmonic in four different liquids are illustrated. Clearly, it is visible that higher viscosities yield higher damping. The shift in the resonance frequencies is not necessarily effected by the mass density but by de-tuning of the sensor (e.g., from thermal stresses). In (b) a comparison between measured data (in this case for isopropanol) and the model obtained by fitting the parameters from Eq. 10 is shown.

\section{References}

[1] C. Tropea et al. , Springer Handbook of Experimental Fluid Mechanics, Springer-Verlag Berlin Heidelberg, 2007

[2] C.A. Nieto de Castro, Metrology of Viscosity: Have We Learned Enough?, J. Chem. Eng. Data 2009, 54, $171-178$

[3] S.J. Martin, V. E. Granstaff and Gregory C. Frye, Characterization of a Quartz Crystal Microbalance with Simultaneous Mass and Liquid Loading, Anal. Chem. 1991, 63, 2272-2281

[4] B. Jakoby, M. Scherer, M. Buskies and H. Eisenschmid, Microacoustic viscosity sensor for automotive applications, Tech. Digest 2002 IEEE Intl. Conf. On Sensors, Orlando, FL, June, 2002, pp. 1587-1590

[5] A. Agoston, C. Ötsch, B. Jakoby, Viscosity sensors for engine oil condition monitoring - Application and interpretation of results, Sensors and Actuators A 121, pp. 327-332, (2005)

[6] E.K. Reichel, Dynamic Methods for Viscosity and Mass-Density Sensing, PhD Thesis, Insitute for Microelectronics and Microsensors, Johannes Kepler University, Linz, Austria 2009

[7] C. Riesch. Micromachined Viscosity Sensors, PhD Thesis, Technical University Vienna, Austria. 2009

[8] M. Heinisch, Electromagnetically Actuated Valveless Micropump in Polymer-Technology for Sensor Applications, Diploma Thesis, Insitute for Microelectronics and Microsensors, Johannes Kepler University, Linz, Austria 2009

[9] G. Habenicht, Kleben, Grundlagen, Technologien, Anwendungen, 6. Auflage, Springer-Verlag Berlin Heidelberg, 2009

[10] C. W. Macosko, Rheology, Principles, Measurements and Applications, Wiley-VCH, 1994

[11] C.J. Seeton, Viscosity-temperature correlation for liquids, Tribology Letters, Vol. 22, No. 1, April 2006

[12] F. Lucklum, E.K. Reichel, B. Jakoby, Miniature Density-Viscosity Measurement Cell Utilizing ElectrodynamicAcoustic Resonator Sensors, Sensors Actuators A (2011), to appear.

[13] M. Kandil, The development of a vibrating wire viscometer and a microwave cavity resonator for the measurement of viscosity, dew points, density, and liquid volume fraction at high temperature and pressure, University of Canterbury, Christchurch, New Zealand 2005

[14] F. Peleties, Advanced Fluid Property Measurement for Oilfield Applications, Department of Chemical Engineering and Chemical Technology Imperial College, London, England 2007 